\title{
Contraste entre las cartas de control MR de Shewhart y Cusum Varianza en el monitoreo del potencial de hidrógeno en protectores de planta
}

\section{Contrast between Shewhart for MR and Cusum Variance control chart in the monitoring of hydrogen potential in plant protectors}

\author{
Roberto José Herrera-Acosta \\ Universidad del Atlántico \\ Barranquilla, Colombia \\ robertoherrera@mail.uniatlantico.edu.co
}

\author{
Indira Dayana Romero-Cabrera \\ Universidad del Atlántico \\ Barranquilla, Colombia \\ idayanaromero@mail.uniatlantico.edu.co
}

\author{
Richard Michael Wasinski-Zuñiga \\ Universidad del Atlántico \\ Barranquilla, Colombia \\ rmwasinski@mail.uniatlantico.edu.co
}

\begin{abstract}
Resumen- Uno de los aspectos más significativos para mejorar la calidad de los procesos es evitar el aumento de la variabilidad, por ello es importante el monitoreo continuo de la característica de calidad, que permita conocer su estado y comportamiento en el tiempo. Una de las principales herramientas son las cartas de control. Hawkins plantea un procedimiento para monitorear la variabilidad del proceso mediante cartas de control CUSUM. En este artículo se presenta su aplicación en la medida de variabilidad de los valores de pH en el uso de fungicidas; así, como los resultados sobre su desempeño en comparación con la carta de control de Shewhart, la cual no es tan efectiva para detectar corrimientos pequeños.
\end{abstract}

Palabras clave- Corrimiento, longitud promedio de corrida, valor de referencia, valor objetivo, varianza.

\begin{abstract}
One of the most significant aspects to improve the quality of the processes is to avoid the increase of the variability, so it is important the continuous monitoring of the quality feature, which allows to know its condition and behavior over time. One of the main tools is Control Charts. Hawkins creates a method for monitoring process variability using the CUSUM Control Charts. This paper shows its application in the measure of variability of the $\mathrm{pH}$ values in the use of fungicides; as well as the results on their performance compared to the Shewhart Control Chart, which is not as effective at detecting small shifts.
\end{abstract}

Keywords- Average run length, reference value, shift, target value, variance.

\section{INTRODUCCIÓN}

Las cartas de control para procesos estadísticos son utilizadas frecuentemente para monitorear una característica de calidad e identificar irregularidades en las medidas de localización y variabilidad aplicadas, que pueden presentarse como cambios repentinos o graduales en los datos de estudio. Comúnmente, en el caso univariado se utilizan las cartas de control Shewhart, CUSUM y EWMA, cuya variación se supone que sigue una distribución normal.

En este artículo se hará una aplicación a un método básico SPC, mediante la utilización de la Carta CUSUM para monitorear la variabilidad del $\mathrm{pH}$ en el uso de fungicidas en la protección de plantas. La carta de CUSUM es una alternativa ante la insensibilidad de los corrimientos pequeños del proceso en las cartas de control de Shewhart, debido a que va acumulando información obtenida de las muestras anteriores y de esta manera puede detectar de forma más rápida pequeños cambios en el proceso [1].

Douglas M. Hawkins [2,3] creó un procedimiento para el estudio de la variabilidad implementando CUSUM. El propósito de este trabajo es aplicar el procedimiento presentado por Hawkins, estudiar la sensibilidad de los datos de un proce- 
so real y comparar los resultados con la carta de control de Shewhart, mediante el cálculo de la longitud de corridas promedio (ARL), con la finalidad de detectar cuál de estas cartas es más sensible a estos cambios.

ARL permite establecer mediante el conteo de puntos, cuál de las cartas de control, en este caso univariado, detecta con más rapidez una señal de alarma, dado un incremento en la variabilidad en el monitoreo de la medida de variabilidad o en la medida de localización de una carta de control.

\section{ESTADO DE ARTE}

\subsection{Fundamentación teórica}

\subsubsection{Principios básicos}

Es el número de puntos graficados en la carta hasta que aparezca una señal fuera de control, cuando el proceso se monitorea en línea, es decir, se encuentra en Fase II, se conoce como longitud de corridas, se donará por RL. La Iongitud de corridas $\mathrm{RL}$ es una variable aleatoria con un comportamiento geométrico de la forma $P(R L=x)=p(1-p)^{x-1}$, con un valor esperado de $A R L=1 / p$, donde en condiciones normales $p=0.0027$, es definida como la probabilidad de que aparezca una señal de alarma. Esta longitud de corridas RL permite para cada una de las cartas de control evaluar su sensibilidad, realizando el conteo de tandas hasta que exista una señal de alarma o punto fuera de los límites; los estudios indican, por ejemplo: mayor potencia en detectar señales de alarma de la carta $S$ en contraste con la carta $\mathrm{R}$ para cualquier incremento de la dispersión. En la carta de control Shewhart, $p$ se define entonces como la probabilidad de que un punto se localice fuera de control, definida como:

$$
\begin{aligned}
& p=p\left(\bar{X}_{i} \leq l c i\right)+p\left(\bar{X}_{i} \geq l c s\right)= \\
& p\left(Z \leq \frac{l c i-\mu}{\sigma / \sqrt{n}}\right)+\left(Z \leq \frac{l c s-\mu}{\sigma / \sqrt{n}}\right)
\end{aligned}
$$

La carta CUSUM fue propuesta por Page (1954), y el nombre de CUSUM se debe a que es una carta en la que se grafican las sumas acumuladas de las desviaciones con respecto al valor nominal de la característica de interés. Por ejemplo, suponer que se tienen muestras de ta- maño $n \geq 1$, y que $x_{j}$ es el promedio de la j-ésima muestra. Entonces, si $\mu_{0}$ es el objetivo para la media del proceso, la carta de control de suma acumulada se construye graficando la cantidad [4]:

$$
C_{i}=\sum_{j=1}^{i}\left(\overline{x_{j}}-\mu_{0}\right)
$$

contra la muestra $i$, el estadístico $C i$ se le llama a la suma acumulada hasta la i-ésima muestra, incluyéndola.

Esta carta constituye una forma rápida de determinar cuánto se aparta una unidad de producto de un estándar de fabricación establecido, determinando el valor aceptable e inaceptable de esta desviación, visualizándose la información en forma de gráfico. Las cartas de control de CUSUM permiten monitorear en forma continua las diferencias acumuladas de una característica cualitativa de un proceso determinado, que puede definirse como datos recogidos secuencialmente para permitir la detección temprana de desviaciones de un estándar establecido $[5,6]$. La principal ventaja de las cartas CUSUM es que puede detectar un cambio pequeño o persistente en el promedio del proceso, más rápidamente que una carta de control Shewhart Tabla 1.

En el campo de los herbicidas o protectores de planta, J.P. Lorente (2012) presenta la importancia de las cartas de control univariadas de Shewhart en la agricultura, afirmando a su vez las grandes dificultades en la implementación en este sector debido al desconocimiento en la aplicación de las herramientas estadísticas en el control de calidad [19].

\subsubsection{Carta de control CUSUM para la varia- bilidad}

Las cartas de control CUSUM son utilizadas para monitorear la medida de variabilidad de un proceso. Supóngase que se desea monitorear la $\sigma^{2}$ de una característica de calidad tomando una muestra de $x_{1}, x_{2}, x_{3}, \ldots, x_{n}$ de $n$ variables independientes. Cuando el proceso está bajo control, tiene una distribución normal con media $\mu_{0}$ y desviación estándar $\sigma$. Se supone que $\sigma$ es conocida o que se cuenta con una estimación de la misma [7].

Para evaluar la variabilidad, Hawkins (1981, 1993) sugiere crear una nueva cantidad estandarizada $[1,3,8]$ :

$$
v_{i}=\frac{\sqrt{\left|y_{i}\right|}-0.822}{0.349}
$$


TABLA I

BREVE EVOLUCIÓN DE LAS CARTAS DE CONTROL

\begin{tabular}{|c|c|c|}
\hline CRONOLOGÍA & CONTEXTUALIZACIÓN & ANÁLISIS \\
\hline $\begin{array}{c}\text { Carta de control } \\
1920\end{array}$ & $\begin{array}{l}\text { Empleado por primera vez por el Dr. Walter } \\
\text { Shewhart en la Bell Telephone. }\end{array}$ & $\begin{array}{l}\text { Utilizada para localizar los problemas ocurridos duran- } \\
\text { te el proceso que puede ser por causas asignables (no } \\
\text { aleatorias) o causas no asignables (aleatorias). }\end{array}$ \\
\hline $\begin{array}{l}\text { Cartas Control } \\
\text { Mutivariante } \\
1947\end{array}$ & $\begin{array}{l}\text { La principal aportación en este campo fue la } \\
\text { realizada por el profesor Harold Hotelling (1947), } \\
\text { quien propuso una carta de control multivariante } \\
\text { basado en la distancia de Mahalanobis (1936). }\end{array}$ & $\begin{array}{l}\text { Las cartas de control multivariante, denominadas } \\
\text { T2 de Hotelling, el cual monitorea la distancia entre } \\
\text { el vector de promedios esperado y el vector de pro- } \\
\text { medios observados, considerando su matriz de va- } \\
\text { rianza covarianzas para evaluar el límite de control. }\end{array}$ \\
\hline $\begin{array}{l}\text { Cartas CUSUM } \\
1954\end{array}$ & $\begin{array}{l}\text { En esta carta se considera el componente de } \\
\text { dependencia en el tiempo de las observaciones } \\
\text { o estadísticos de control, introducido por E. S. } \\
\text { Page (1954). }\end{array}$ & $\begin{array}{l}\text { Estas cartas van acumulando información obtenida } \\
\text { de las muestras anteriores; de esta forma pueden } \\
\text { detectar con rapidez cambios pequeños en el pro- } \\
\text { ceso. }\end{array}$ \\
\hline $\begin{array}{l}\text { Carta Ewma } \\
1959\end{array}$ & $\begin{array}{l}\text { S. W. Roberts (1959) denominado carta de pro- } \\
\text { medios móviles exponencialmente ponderados } \\
\text { (EWMA). }\end{array}$ & $\begin{array}{l}\text { Aplica como estadístico de control una ponderación } \\
\text { asociada a las observaciones anteriores, para sen- } \\
\text { sibilizar la carta cuando los cambios son pequeños. }\end{array}$ \\
\hline $\begin{array}{l}\text { CUSUM R } \\
1963\end{array}$ & E.S. Page adopta la estadística de control R. & $\begin{array}{l}\text { Introduce el estadístico de control del rango en las } \\
\text { sumas acumuladas. }\end{array}$ \\
\hline $\begin{array}{l}\text { CP CUSUM } \\
1999\end{array}$ & $\begin{array}{l}\text { Acosta, Mejía, Pignatello y Reo utilizan la trans- } \\
\text { formación normal inversa para determinar el es- } \\
\text { tadístico de la carta CUSUM [17]. }\end{array}$ & $\begin{array}{l}\text { Para monitorear la variabilidad tomando como base } \\
\text { la razón de verosimilitud. }\end{array}$ \\
\hline $\begin{array}{c}\text { Carta MCUSUM } \\
1988\end{array}$ & $\begin{array}{l}\text { Crosier presentó una versión multivariada de la } \\
\text { carta CUSUM. }\end{array}$ & $\begin{array}{l}\text { Las cartas multivariantes están sujetas al supuesto de } \\
\text { normalidad multivariada que podría ser una limitación } \\
\text { para los casos donde se rechace esta hipótesis. }\end{array}$ \\
\hline $\begin{array}{l}\text { Carta Mewma } \\
1992\end{array}$ & $\begin{array}{l}\text { Lowry a través de la extensión de la carta de } \\
\text { control EWMA para el caso multivariante. }\end{array}$ & \\
\hline $\begin{array}{l}\text { CUSUM V } \\
2002\end{array}$ & $\begin{array}{l}\text { Petrodjo, Abdollahian y Narayan definen un esta- } \\
\text { dístico de control Vt aproximado a una distribu- } \\
\text { ción chi-cuadrado[18]. }\end{array}$ & $\begin{array}{l}\text { Esta carta es más sensible que la carta CUSUM } \\
\text { para la desviación } \mathrm{S} \text { y el rango R. }\end{array}$ \\
\hline DDChart & $\begin{array}{l}\text { R. Liu aporta el diseño de la carta de control de- } \\
\text { nominada cartas DDChart, }\end{array}$ & $\begin{array}{l}\text { Cartas de control de tipo no paramétrico, basados } \\
\text { en el concepto de profundidad de datos. }\end{array}$ \\
\hline
\end{tabular}

Fuente: Los autores.

Donde $y_{i}$ es el valor estandarizado de la variable aleatoria $x_{i}$, y está dado por:

$$
y_{i}=\frac{x_{i}-\mu_{0}}{\sigma}
$$

Hawkins sugiere que las $v_{i}$ son más sensibles a los corrimientos en la varianza que a los de la media. Puesto que la distribución bajo control de $v_{i}$ es aproximadamente $N \sim(0,1)$, es posible establecer los siguientes CUSUM unilaterales (estadísticos de control) a escala estandarizada [9]:

$$
\begin{aligned}
& S_{i}^{+}=\max \left[0, v_{i}-k+S_{i-1}^{+}\right] \\
& S_{i}^{-}=\max \left[0,-k-v_{i}+S_{i-1}^{-}\right] \\
& \text {donde: } S_{0}^{+}=S_{0}^{-}=0
\end{aligned}
$$

Para la aplicación de esta carta, es importante definir dos parámetros. El primero de ellos corres- ponde a $K$, conocido como valor de referencia (o tolerancia, o valor laxo), el cual determina la sensibilidad del gráfico y se obtiene como $K=k \sigma$. La elección de $k$ depende del tamaño del corrimiento que quiere detectarse; es decir, $k=\frac{1}{2} \delta$ donde $\delta$ es la magnitud del corrimiento en unidades de desviación estándar [10].

Otro parámetro que se debe considerar es el intervalo de decisión $H$, criterio para decidir si el proceso está fuera de control. Se define $H=h \sigma$, donde $\sigma$ es la desviación estándar de la variable muestral usada para formar la CUSUM. Por lo general, este valor suele ser cinco veces la desviación estándar del proceso $\sigma$. Cabe anotar, que diversos estudios analíticos [3] recomiendan utilizar $h=4$ o $h=5$ y $k=0,5$, ya que con es- 
tos valores se alcanzará una CUSUM que tiene un buen desempeño de la longitud promedio de la corrida (ARL).

En la Tabla II se muestra las longitudes promedio de las corridas bilaterales para $h=4 \mathrm{y}$ $h=5$ con $k=0,5$ Para la carta de control, se debe tener en cuenta que si la desviación estándar del proceso aumenta, los valores de $S_{i}^{+}$igualmente aumentarán y terminaran por exceder el valor de $h$; por otro lado, si la desviación estándar disminuye, entonces los valores de $S_{i}^{-}$aumentarán y terminarán por exceder $-h$.

$$
\begin{gathered}
\text { TABLA II } \\
\text { DESEMPEÑO DE LA ARL DE LA CUSUM TABULAR CON } k=\frac{1}{2} \text { Y } \\
h=4 \text { o } h=5
\end{gathered}
$$

\begin{tabular}{|c|c|c|}
\hline $\begin{array}{c}\text { Corrimiento en la media } \\
\text { (múltiplo de s) }\end{array}$ & $\mathrm{h}=4$ & $\mathrm{~h}=5$ \\
\hline 0.00 & 168 & 465 \\
\hline 0.25 & 74.2 & 139 \\
\hline 0.50 & 26.6 & 38.0 \\
\hline 0.75 & 13.3 & 17,0 \\
\hline 1.00 & 8.38 & 10.4 \\
\hline 1.50 & 4.75 & 5.75 \\
\hline 2.00 & 3.34 & 4.01 \\
\hline 2.50 & 2.62 & 3.11 \\
\hline 3.00 & 2.19 & 2.57 \\
\hline 4.00 & 1.71 & 2.01 \\
\hline
\end{tabular}

Fuente: D.C. Montgomery, Introduction to Statical Quality Control.

En estos casos, si $S_{i}^{+}$o $S_{i}^{-}$excede el intervalo de decisión $H$, se considera que el proceso está fuera de control, deberán buscarse las causas asignables que generaron este hecho y emprender cualquier acción correctiva.

Los valores de $\mathrm{N}+$ y $\mathrm{N}$ - indican los períodos en que las sumas no han sido cero, en la carta CUSUM permite conocer cuando ha ocurrido el corrimiento. De esta manera permite contar hacia atrás desde un punto fuera de control hasta el período de tiempo en que la CUSUM subió de cero, para encontrar el primer período del proceso posterior al corrimiento.

Los cálculos de los ARL de la Tabla II se verificaron por simulación fijando una longitud promedio de corrida nominal o primaria $A R L 0=200$, estableciendo el límite unilateral superior de control para la carta e intervalo de muestreo fijo. Se supuso que los esquemas trabajan en las condiciones de Fase II de control. El proceso de simulación se llevó a cabo mediante el programa de computación diseñado en lenguaje $\mathrm{R}$, el cual compara simultáneamente para los valores de $h$ cuatro (4) y (5). El algoritmo que se aplicó es el siguiente:

a) Inicialmente se evaluó el límite de control superior de referencia en cada uno de los esquemas, bajo un valor nominal ARL igual a 200, para los valores de $h$ de cuatro y cinco. b) Una vez establecido el valor del límite superior de control, se generan los subgrupos cuyas observaciones provienen de una distribución normal con media y desviación conocida y se generaron grupos de observaciones provenientes de una distribución normal con desviación estándar modificada. c) Para cada muestra se calculó del estadístico de control para la variabilidad. d) Posteriormente en cada uno de los esquemas se compara su valor con el límite superior respectivo. Cada vez que el estadístico excedía al valor del límite superior respectivo, se detiene la simulación; de lo contrario, el proceso continuaba. e) Los pasos anteriores se replicaron 15000 veces, efectuando 1000 veces las mismas réplicas y los resultados obtenidos en cada iteración para un mismo esquema de control se promediaron, obteniendo así la longitud media de corridas ARL.

\subsubsection{Carta de Shewhart para observacio- nes individuales}

Con el fin de estimar la variabilidad debida a causas especiales cuando se presentan lecturas individuales que constituyen tendencias, Shewhart presenta la carta de control de observaciones individuales En esta carta se selecciona una observación por subgrupo de la característica de calidad de interés; y se monitorea la variabilidad mediante el rango móvil (MR).

Los límites históricos para el rango móvil están definidos como [9]

$$
\begin{aligned}
& U C L=D_{4}(2) \bar{M} R C L= \\
& \bar{M} R L C L=D_{3}(2) \bar{M} R
\end{aligned}
$$

Los límites para la medida de localización en la Fase I, están dados por: 


$$
\begin{aligned}
& U C L=\bar{X}+3\left(\bar{M} R / d_{2}(2)\right) C L= \\
& \bar{X} L C L=\bar{X}-3\left(\overline{M R} / d_{2}(2)\right)
\end{aligned}
$$

vil

donde la media es $\bar{X}=\frac{\sum_{i=1}^{m} X_{i}}{m}$ y el rango mó-

$$
\bar{M} R=\frac{\sum_{l=1}^{n}\left|X_{i}-X_{1-i}\right|}{n-1}
$$

En esta primera parte se asume el desconocimiento de los parámetros de control $(\sigma, \mu)$. Cuando se presenta un hecho no aleatorio o asignable, los responsables del proceso deben encontrar una causa atribuible realizando las correcciones pertinentes para que esta situación no se presente. Luego, se debe eliminar la señal fuera de control, para determinar posteriormente los parámetros del proceso.

$$
E(\bar{M} R)=\frac{\bar{M} R}{d_{2}(2)}=\sigma E(\bar{X})=\mu
$$

Subsiguientemente, en la Fase II la formulación de los límites de control para la medida de variabilidad están dados como:

$$
\begin{aligned}
& U C L=D_{2}(2) \sigma C L=d_{2}(2) \sigma L C L \\
& =D_{1}(2) \sigma
\end{aligned}
$$

La formulación para los límites estándar de control de la medida de localización, Fase II son en su orden:

$$
U C L=\mu+3 \sigma C L=\mu L C L=\mu-3 \sigma
$$

\section{APLICACIÓN EN EL USO DE FUNGICIDAS}

Las plantas como cualquier ser vivo son susceptibles de enfermarse, estas enfermedades son las principales fuentes de daño de plantas y cultivos, causadas primeramente por organismos fitopatógenos. Siendo los hongos la principal causa de pérdida de cultivos a nivel mundial. En este sentido, se hace indispensable manejar adecuadamente las enfermedades de las plantas bajo un riguroso control que encierra desde la rotación de cultivos, selección de cultivares tolerantes o resistentes, época de siembra, nivel de fertilización, modificación del microclima, sanidad hasta la aplicación de fungicidas.

En este artículo se explicará qué son los fungicidas, por qué son necesarios, y la calidad de la mezcla fungicida; en esta última se resaltará la calidad del agua en función del pH y su efecto sobre la estabilidad y eficacia del fungicida. La importancia de este estudio es introducir en esta área las herramientas del control estadístico de proceso CEP, en la evaluación, monitoreo y análisis de la información mediante técnicas gráficas, como son las cartas de control, específicamente la carta CUSUM debido a su sensibilidad a pequeños cambios, por lo que se hace indispensable, en este caso, en la evaluación de características de calidad como el pH del fungicida.

A fin de comprender la importancia del $\mathrm{pH}$ en la estabilidad y eficacia de un fungicida, en un comienzo, es transcendental abordar el concepto de fungicida.

En este sentido, según Whindham (2016), un fungicida es un "Compuesto que mata o inhibe el crecimiento de hongos" [12]. Igualmente, en [13] se define a un fungicida como "un tipo particular de plaguicida que controla enfermedades fúngicas, inhibiendo o eliminando al hongo que causa la enfermedad". Por otra parte, otros autores definen a un fungicida como "todos aquellos compuestos que pueden proporcionar resistencia a la planta huésped o que convierten el medio ambiente en un lugar inadecuado para el desarrollo y crecimiento del organismo infeccioso" [14].

En un sentido general, existen tres razones principales por las cuales se utilizan los fungicidas:

a. Para controlar a la enfermedad durante el establecimiento y desarrollo de un cultivo.

b. Para incrementar la productividad de un cultivo y reducir sus daños.

c. Para mejorar el período de almacenamiento y la calidad de las plantas y los productos cosechados.

A lo que respecta a la calidad de la mezcla fungicida, se debe hacer hincapié a la preparación de la mezcla, ya que este aspecto es fundamental para asegurar la correcta distribución y estabilidad de los componentes por unidad de volumen. "A lo anterior debe sumarse la calidad del agua (esto en el caso de preparaciones en agua o en emulsión). Las características químicas del agua ( $\mathrm{pH}$ y dureza, principalmente) pue- 
den afectar la efectividad del (los) ingrediente (s) activo (s)" [15].

La mayoría de los fungicidas, particularmente los organofosforados y los carbamatos atraviesan una reacción química en presencia de componentes alcalinos que destruyen su efectividad. Esta reacción es llamada hidrólisis alcalina y ocurre cuando el pesticida es mezclado con agua alcalina (con un pH mayor a 7). A mayor alcalinidad del agua, más rápido se desvanece la efectividad de los mismos. Por ejemplo, en un fungicida " $X$ ", el tiempo para que ocurra el $50 \%$ de la hidrólisis a pH 8.0 es 63 minutos; a pH 7.0 ocurre en 386 minutos y a pH 6.0, 80 horas. Esto significa que si el $\mathrm{pH}$ de su agua es 8.0 y pasa una hora desde que agregó el fungicida al tanque y lo aplicó, el $50 \%$ de la materia activa ya está descompuesta. Pero si el agua tiene un pH 6.0, no perderá una cantidad significativa durante la aplicación [16].

En definitiva, el grado de destrucción de una mezcla fungicida varía de acuerdo con la alcalinidad y la temperatura del agua y el tiempo en que la mezcla permanece en el tanque. Es aquí, donde radica la importancia de las mediciones de $\mathrm{pH}$, ca- racterística que se ha tomado como ejemplo para este artículo, ya que permite asegurar que dicha hidrólisis pase lo más lento posible y asimismo asegurar la estabilidad y eficacia del producto. Cabe resaltar que estos valores de $\mathrm{pH}$ han sido modificados por una constante.

\section{RESULTADOS}

\subsection{Aplicación carta CUSUM}

En la Tabla III se presenta una muestra $x_{i}$ de la característica de calidad de interés (valores de $\mathrm{pH})$, con $\mu=14,6520$ y $\sigma=0,4329$. Igualmente se exhibe la cantidad estandarizada $v_{i}$ de cada muestra, obtenida mediante la ecuación (2), los estadísticos de control $S_{i}^{+}$y $S_{i}^{-}$, hallados con las fórmulas (4) y (5) respectivamente y los contadores $N^{+}$y $N^{-}$.

Con el fin de producir un buen desempeño de la ARL se supondrá que la magnitud del corrimiento que quiere detectarse es de $\delta=1$; por lo tanto, se define un valor de sensibilidad de la carta o referencia de $k=0,5$ y además, se

TABLA III

INFORMACIÓN DE LA MEDICIÓN DEL pH Y APLICACIÓN CARTA CUSUM

\begin{tabular}{|c|c|c|c|c|c|c|c|}
\hline Número del subgrupo & $\mathrm{X}_{\mathrm{i}}$ & $\mathrm{Y}_{\mathrm{i}}$ & $\mathrm{V}_{\mathrm{i}}$ & $\mathrm{Si}^{+}$ & $\mathrm{N}^{+}$ & $\mathrm{Si}^{-}$ & $\mathrm{N}^{-}$ \\
\hline 1 & 14,790 & 0,319 & $-0,738$ & 0,000 & 0,000 & 0,238 & 1,000 \\
\hline 2 & 14,640 & $-0,028$ & $-1,878$ & 0,000 & 0,000 & 1,616 & 2,000 \\
\hline 3 & 14,730 & 0,180 & $-1,139$ & 0,000 & 0,000 & 2,255 & 3,000 \\
\hline 4 & 14,160 & $-1,137$ & 0,699 & 0,199 & 1,000 & 1,055 & 4,000 \\
\hline 5 & 14,970 & 0,735 & 0,101 & 0,000 & 0,000 & 0,455 & 5,000 \\
\hline 6 & 14,625 & $-0,062$ & $-1,640$ & 0,000 & 0,000 & 1,595 & 6,000 \\
\hline 7 & 15,660 & 2,329 & 2,017 & 1,517 & 1,000 & 0,000 & 0,000 \\
\hline 8 & 14,025 & $-1,448$ & 1,093 & 2,110 & 2,000 & 0,000 & 0,000 \\
\hline 9 & 15,030 & 0,873 & 0,322 & 1,932 & 3,000 & 0,000 & 0,000 \\
\hline 10 & 14,340 & $-0,721$ & 0,077 & 1,510 & 4,000 & 0,000 & 0,000 \\
\hline 11 & 14,700 & 0,111 & $-1,401$ & 0,000 & 0,000 & 0,901 & 1,000 \\
\hline 12 & 14,190 & $-1,067$ & 0,605 & 0,105 & 1,000 & 0,000 & 0,000 \\
\hline 13 & 15,030 & 0,873 & 0,322 & 0,000 & 0,000 & 0,000 & 0,000 \\
\hline 14 & 14,640 & $-0,028$ & $-1,878$ & 0,000 & 0,000 & 1,378 & 1,000 \\
\hline 15 & 14,640 & $-0,028$ & $-1,878$ & 0,000 & 0,000 & 2,757 & 2,000 \\
\hline 16 & 14,325 & $-0,755$ & 0,135 & 0,000 & 0,000 & 2,122 & 3,000 \\
\hline 17 & 15,435 & 1,809 & 1,498 & 0,998 & 1,000 & 0,123 & 4,000 \\
\hline 18 & 14,475 & $-0,409$ & $-0,523$ & 0,000 & 0,000 & 0,146 & 5,000 \\
\hline 19 & 14,640 & $-0,028$ & $-1,878$ & 0,000 & 0,000 & 1,525 & 6,000 \\
\hline 20 & 13,995 & $-1,518$ & 1,175 & 0,675 & 1,000 & 0,000 & 0,000 \\
\hline
\end{tabular}

Fuente: Autores. 
utiliza un intervalo de decisión de cinco veces la desviación estándar del proceso $\sigma$, es decir, $U C L=5(0,4329)=2,1645, C L=0,4329 y$

$L C L=-5(0,4329)=-2,1645$

Al graficar los estadísticos de control $S_{i}^{+}$y $S_{i}^{-}$se obtienen dos puntos fuera de control, como se muestra en la Fig. 1. Estos puntos fuera de los límites poseen una causa asignable; por lo tanto, son eliminados.

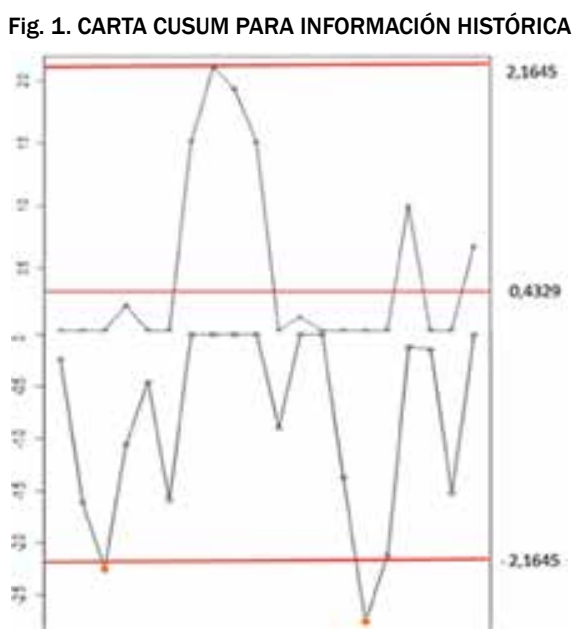

Se procede de nuevo a evaluar y graficar los estadísticos de control para los datos restantes, como se observa Tabla IV.

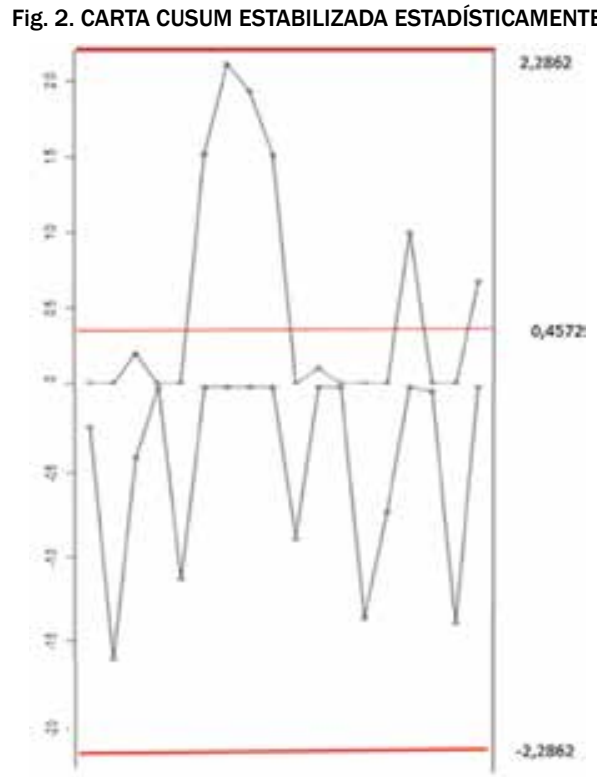

Fuente: Autores

El proceso se encuentra en un estado de estabilidad estadística con parámetros $\mu=14,6483$ y

Fuente: Autores.

TABLA IV

APLICACIÓN CARTA CUSUM PARA LOS SUBGRUPOS RESTANTES

\begin{tabular}{|c|c|c|c|c|c|c|c|}
\hline Número del subgrupo & $\mathrm{X}_{\mathrm{i}}$ & $\mathrm{Y}_{\mathrm{i}}$ & $\mathrm{V}_{\mathrm{i}}$ & $\mathrm{Si}^{+}$ & $\mathrm{N}^{+}$ & $\mathrm{Si}^{-}$ & $\mathrm{N}^{-}$ \\
\hline 1 & 14,790 & 0,319 & $-0,738$ & 0,000 & 0,000 & 0,238 & 1,000 \\
\hline 2 & 14,640 & $-0,028$ & $-1,878$ & 0,000 & 0,000 & 1,616 & 2,000 \\
\hline 4 & 14,160 & $-1,137$ & 0,699 & 0,199 & 1,000 & 0,416 & 3,000 \\
\hline 5 & 14,970 & 0,735 & 0,101 & 0,000 & 0,000 & 0,000 & 0,000 \\
\hline 6 & 14,625 & $-0,062$ & $-1,640$ & 0,000 & 0,000 & 1,140 & 1,000 \\
\hline 7 & 15,660 & 2,329 & 2,017 & 1,517 & 1,000 & 0,000 & 0,000 \\
\hline 8 & 14,025 & $-1,448$ & 1,093 & 2,110 & 2,000 & 0,000 & 0,000 \\
\hline 9 & 15,030 & 0,873 & 0,322 & 1,932 & 3,000 & 0,000 & 0,000 \\
\hline 10 & 14,340 & $-0,721$ & 0,077 & 1,510 & 4,000 & 0,000 & 0,000 \\
\hline 11 & 14,700 & 0,111 & $-1,401$ & 0,000 & 0,000 & 0,901 & 1,000 \\
\hline 12 & 14,190 & $-1,067$ & 0,605 & 0,105 & 1,000 & 0,000 & 0,000 \\
\hline 13 & 15,030 & 0,873 & 0,322 & 0,000 & 0,000 & 0,000 & 0,000 \\
\hline 14 & 14,640 & $-0,028$ & $-1,878$ & 0,000 & 0,000 & 1,378 & 1,000 \\
\hline 16 & 14,325 & $-0,755$ & 0,135 & 0,000 & 0,000 & 0,743 & 2,000 \\
\hline 17 & 15,435 & 1,809 & 1,498 & 0,998 & 1,000 & 0,000 & 0,000 \\
\hline 18 & 14,475 & $-0,409$ & $-0,523$ & 0,000 & 0,000 & 0,023 & 1,000 \\
\hline 19 & 14,640 & $-0,028$ & $-1,878$ & 0,000 & 0,000 & 1,401 & 2,000 \\
\hline 20 & 13,995 & $-1,518$ & 1,175 & 0,675 & 1,000 & 0,000 & 0,000 \\
\hline
\end{tabular}

Fuente: Autores. 
$\sigma=0,4572$. Los límites de control están dadospor $U C L=5(0,4572)=2,2862, C L=0,4572$ y $L C L=-5(0,4572)=-2,2862$.
Finalmente, se hace control a la nueva información seleccionada del proceso; tomando como referente estos límites estandarizados, Tabla V.

TABLA V

APLICACIÓN CARTA CUSUM A LOS DATOS DE CONTROL

\begin{tabular}{|c|c|c|c|c|c|c|c|}
\hline Número del subgrupo & $\mathrm{X}_{i}$ & $\mathrm{Y}_{i}$ & $\mathrm{~V}_{i}$ & $\mathrm{~S}_{i}^{+}$ & $\mathrm{N}^{+}$ & $\mathrm{Si}^{-}$ & $\mathrm{N}^{-}$ \\
\hline 1 & 15,720 & 2,344 & 2,031 & 1,531 & 1,000 & 0,000 & 0,000 \\
\hline 2 & 15,270 & 1,360 & 0,986 & 2,017 & 2,000 & 0,000 & 0,000 \\
\hline 3 & 15,075 & 0,933 & 0,413 & 1,930 & 3,000 & 0,000 & 0,000 \\
\hline 4 & 15,600 & 2,081 & 1,778 & 3,208 & 4,000 & 0,000 & 0,000 \\
\hline 5 & 15,000 & 0,769 & 0,158 & 2,866 & 5,000 & 0,000 & 0,000 \\
\hline 6 & 14,745 & 0,211 & $-1,038$ & 1,328 & 6,000 & 0,538 & 1,000 \\
\hline 7 & 14,775 & 0,277 & $-0,847$ & 0,000 & 0,000 & 0,885 & 2,000 \\
\hline 8 & 14,715 & 0,146 & $-1,261$ & 0,000 & 0,000 & 1,646 & 3,000 \\
\hline 9 & 14,715 & 0,146 & $-1,261$ & 0,000 & 0,000 & 2,407 & 4,000 \\
\hline 10 & 14,115 & $-1,166$ & 0,739 & 0,239 & 1,000 & 1,168 & 5,000 \\
\hline 11 & 14,490 & $-0,346$ & $-0,669$ & 0,000 & 0,000 & 1,337 & 6,000 \\
\hline 12 & 14,715 & 0,146 & $-1,261$ & 0,000 & 0,000 & 2,099 & 7,000 \\
\hline
\end{tabular}

Fuente: Autores.

En la Fig. 3 se muestra la carta de control CUSUM para estos datos sometidos a control.

Fig. 3. CARTA CUSUM APLICADA A DATOS DE CONTROL

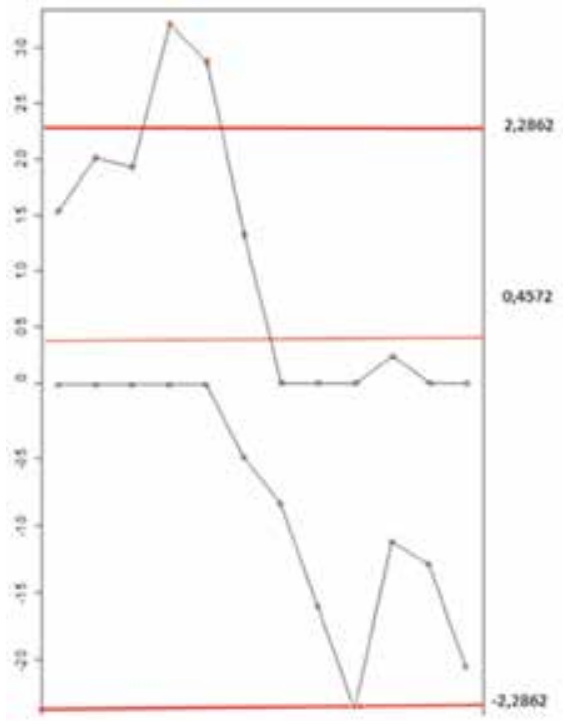

Fuente: Autores.

\subsection{Aplicación carta SHEWHART}

En la Tabla VI se presenta la información histórica de la característica de calidad $x_{i}$. Así, como los valores de MR para los 20 subgrupos.
TABLA VI

INFORMACIÓN DE LA MEDICIÓN DE pH Y APLICACIÓN CARTA SHEWHART

\begin{tabular}{|c|c|c|}
\hline Número del Subgrupo & $X_{i}$ & MR \\
\hline 1 & 14,790 & \\
\hline 2 & 14,640 & 0,150 \\
\hline 3 & 14,730 & 0,090 \\
\hline 4 & 14,160 & 0,570 \\
\hline 5 & 14,970 & 0,810 \\
\hline 6 & 14,625 & 0,345 \\
\hline 7 & 15,660 & 1,035 \\
\hline 8 & 14,025 & 1,635 \\
\hline 9 & 15,030 & 1,005 \\
\hline 10 & 14,340 & 0,690 \\
\hline 11 & 14,700 & 0,360 \\
\hline 12 & 14,190 & 0,510 \\
\hline 13 & 15,030 & 0,840 \\
\hline 14 & 14,640 & 0,390 \\
\hline 15 & 14,640 & 0,000 \\
\hline 16 & 14,325 & 0,315 \\
\hline 17 & 15,435 & 1,110 \\
\hline 18 & 14,475 & 0,960 \\
\hline 19 & 14,640 & 0,165 \\
\hline 20 & 13,995 & 0,645 \\
\hline
\end{tabular}

Fuente: Autores.

Al aplicar la fórmula (6), se obtienen los límites de control históricos para el rango móvil. Dados por: 
$U C L=(3,267)(0,6118)=1,9989, C L=$

0,6118 y $L C L=(0)(0,6118)=0$

Fig. 4. CARTA DE CONTROL SHEWHART PARA EL RANGO MÓVIL

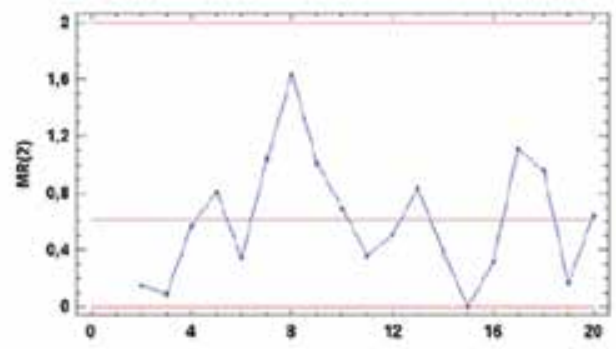

Fuente: Autores.

Igualmente, se hallan los límites de control histórico para la media, definido en la ecuación (7) como

$$
\begin{aligned}
& U C L=14,652+3\left(\frac{0,6118}{1,128}\right)= \\
& 16,2792, C L=14,6520 y L C L \\
& =4,652-3\left(\frac{0,6118}{1,128}\right)=13,0248
\end{aligned}
$$

Fig. 5. CARTA DE CONTROL SHEWHART PARA LA MEDIA

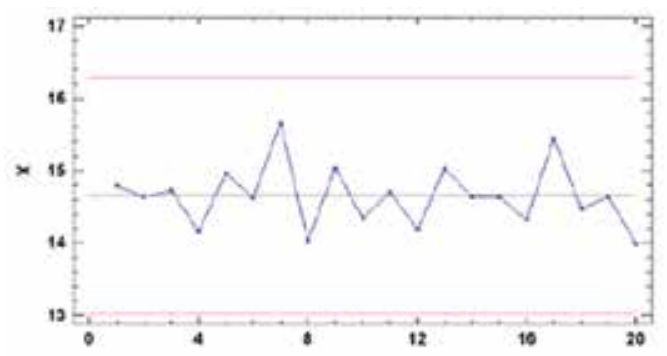

Fuente: Autores.

El proceso se encuentra estabilizado estadísticamente; así que, se estiman los parámetros con la fórmula (8) $\sigma=0,5424 y \mu=14,6520$. El siguiente paso es hacer control con los datos de la Tabla VII.

Primeramente, se evalúan los límites estándar del rango móvil, correspondientes a la ecuación (9)

$$
\begin{aligned}
& C L=(3,686)(0,5424)=1,9993, \\
& L C=(1,128)(0,5424)=0,6118 \\
& y L C L=(0)(0,5424)=0
\end{aligned}
$$

TABLA VII

APLICACIÓN CARTA SHEWHART A LOS DATOS DE CONTROL

\begin{tabular}{|c|c|c|}
\hline Número del Subgrupo & $\mathrm{Xi}$ & $\mathrm{MR}$ \\
\hline 1 & 15,720 & \\
\hline 2 & 15,270 & 0,450 \\
\hline 3 & 15,075 & 0,195 \\
\hline 4 & 15,600 & 0,525 \\
\hline 5 & 15,000 & 0,600 \\
\hline 6 & 14,745 & 0,255 \\
\hline 7 & 14,775 & 0,030 \\
\hline 8 & 14,715 & 0,060 \\
\hline 9 & 14,715 & 0,000 \\
\hline 10 & 14,115 & 0,600 \\
\hline 11 & 14,490 & 0,375 \\
\hline 12 & 14,715 & 0,225 \\
\hline & & \\
\hline
\end{tabular}

Fuente: Autores.

Fig. 6. CARTA DE SHEWHART PARA EL RANGO MÓVIL DE LOS DATOS SOMETIDOS A CONTROL

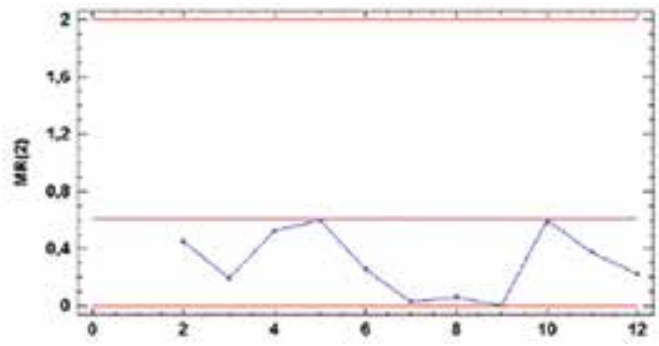

Fuente: Autores

Seguidamente, se hallan los límites estándar de la media, definidos por la ecuación (10), como,

$$
\begin{aligned}
& U C L=16,2792, C L= \\
& 14,6520 \text { y } L C L=13,0248
\end{aligned}
$$

Fig. 7. CARTA DE SHEWHART PARA LA MEDIA DE LOS DATOS SOMETIDOS A CONTROL

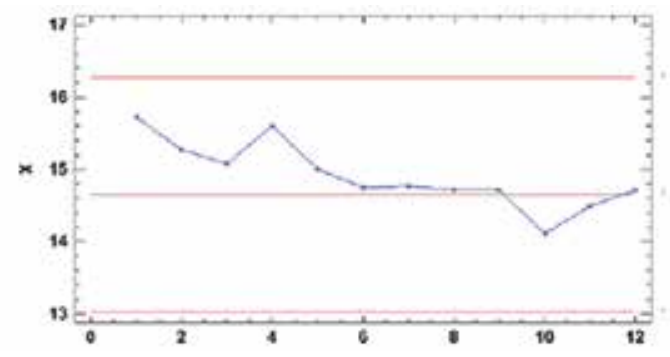

Fuente: Autores.

\section{ANÁLISIS DE LOS RESULTADOS}

Es importante resaltar el comportamiento de las cartas Shewhart y CUSUM en la Fase I de estabilización estadística o estudio inicial. En el caso 
de carta MR de Shewhart, específicamente la medida de variabilidad, el comportamiento sugiere una variabilidad que permite asumir una estabilización de la carta; comportamiento de estabilización más diciente en la carta de la medida de localización, donde la aleatorización alrededor de la media es evidente.

Se puede indicar que para la carta tradicional de Shewhart MR, la estimación de los parámetros es fácil de establecer. El caso de la carta univariada CUSUM difiere en forma importante bajo la misma información, obsérvese en la carta, Fig. 2, a pesar de eliminar los subgrupos que presentan señales de alarma debido a causas asignables, detectadas en el monitoreo del proceso; el comportamiento de las tandas indican una gran variabilidad debido a que existen puntos cercanos a los límites de control establecidos. Es decir, la estabilización de la carta es compleja, permite señalar de esta forma un proceso que requiere de intervención para lograr una mejor estabilización.

En la Fase II o Fase de control, de acuerdo con la Tabla II, un corrimiento de una desviación se detectaría en 10.4 muestras para la carta CUSUM, en comparación con la carta de control de Shewhart para mediciones individuales MR que requeriría 43.96 subgrupos o puntos, en promedio, para detectar ese corrimiento. Es decir, la potencia de la carta CUSUM en detectar cambios pequeños en el proceso, permite sugerir en el caso práctico de estudio, una gráfica acertada a las condiciones del proceso.

Esto se demuestra con la información sometida a control, la carta Shewhart MR, no detecta a lo largo del proceso señales de alarma o fuera de control, por el contrario, sugiere un mejoramiento en el proceso al disminuir la variabilidad de este, Fig. 6. Mientras que la carta CUSUM muestra tres (3) puntos fuera de control para los primeros subgrupos, mejor sensibilidad al detectar más señales de alarma en contraste con la carta MR de Shewhart, como también una tendencia decreciente pronunciada; lo que indica un estado fuera de control denominado cambio de nivel. Esta tendencia también es detectada en la carta Shewhart MR pero en forma suavizada. Esta mejor potencia en detectar señales de alarma de la carta CUSUM, se debe a que combina información de varias muestras y por lo tanto, es más eficien- te al detectar cambios pequeños de la medida de variabilidad.

\section{CONCLUSIONES}

La toma de decisiones para el mejoramiento de los procesos se basa en la información generada por las cartas de control. Así, una carta de control deberá ser capaz de detectar la magnitud del cambio que ocurre en el proceso, con la intención de realizar las mejoras o cambios, que realmente el proceso requiera. En este artículo se aplicó la carta CUSUM para la medida de variabilidad en una muestra de potencial de hidrógeno $\mathrm{pH}$, en protectores de plantas.

El estudio comparó la carta tradicional MR de Shewhart, con respecto a la CUSUM: se observó en esta última, una longitud de corrida promedio ARL menor que la carta de Shewhart. Esto indica que la propuesta CUSUM varianza posee mayor sensibilidad en detectar señales de alarma que la carta tradicional de Shewhart para observaciones individuales.

\section{REFERENCIAS}

[1] A.D. Karaoglan, y G.M. Bayhan, "ARL performance of residual control charts for trend AR (1) process: A case study on peroxide values of stored vegetable oil," Scientific Research and Essays, vol. 7 no.13, pp. 1405-1414, 9 abril 2012.

[2] D.M, Hawkins, "A CUSUM for a scale parameter," Journal of Quality Technology, vol. 13, no. 4, pp. 228-231, 1981.

[3] D.M, Hawkins, "Cumulative Sum Control Charting: An Underutilized SPC Tool," Quality Engineering, vol. 15, no. 3, pp. 463-477, 1993.

[4] V.V Koshti, "Cumulative sum control chart", International Journal of Physics and Mathematical Sciences, vol. 1, no. 1, pp. 28-32, octubre-diciembre 2013.

[5] O.L Davies, and P.L Goldsmith, "Statistical methods in reaserch and production," John Wiley \& Sons, London, May. 1986.

[6] O. L. Davies y P. Royston, "The hidden effects of time," Statistics in Medicine. vol. 7, no. 6, pp. 629-637, junio 1988.

[7] R. J. Herrera, A.A. Mendoza y T. Fontalvo, "Comparación de las Cartas de Control Univariadas con Transformación en la Medida de Variabilidad," INGENIARE, vol. 13, pp. 51-57, octubre 2012.

[8] E.C. Villanueva, "Carta de control No-paramétrica de punto de cambio para varianza," Tesis M.Sc., Facultad Ciencias Físico-matemáticas, Univ. Autónoma de Nuevo León, Monterrey, 2013.

[9] D.C. Montgomery, "Control estadístico de la calidad," Limusa, 3a Edición, ciudad de México, México, pp. 406-424, 1995. 
[10] W. Zhang, y M. Yang, "A CUSUM chart using absolute sample values to monitor process mean and variance," Industrial Engineering and Engineering Management, IEEE conference, 2009.

[11] R. J. Herrera, "Seis Sigma Métodos Estadísticos y sus Aplicaciones," Ecoe Ediciones, pp. 56. 2006.

[12] FRAC (Fungicide resistance action committee), "Clasificación de fungicidas," 2016. [En línea]. Disponible en: https://www.queaplico.com/biblioteca?id=7

[13] M.T. McGrath, “¿Qué son los fungicidas?,” The Plant Health Instructor. Trans. L. Santamaría, J.C Ureta, Cornell University. 2014. [En línea]. Disponible en: http:// www.apsnet.org/EDCENTER/INTROPP/TOPICS/Pages/fungicidesSpanish.aspx

[14] J. Melgarejo y F. Abella, "Mecanismo de acción de los fungicidas," Revista Ventana al Campo, pp. 193-202. 2011.

[15] I. Martínez y M. Guzmán, “Guía básica para la preparación de mezclas, uso de fungicidas y calibración de motobombas utilizadas en el combate de la Sigatoka negra," Dirección de Investigaciones Sección de Fitopatología, no. 3, febrero 2011.
[16] W. K. Hock, "Effect of pH on pesticide stability and efficacy," Pesticide Safety Education Program (PSEP). 2012. [En línea]. Cornell University. Disponible en: http://psep.cce.cornell.edu/facts-slides-self/facts/ gen-peapp-ph.aspx.

[17] C. Acosta, J. Mejía, J. Pignatello, and V. Reo, "A comparation of control charting procedures for monitoring process dispersion," IIE Transactions, vol. 31, pp. 569579, 1999

[18] E.S. Page, "Controlling the standard deviation by CUSUM and warning lines," Technometrics, vol. 5 pp. 307, 1963.

[19] J.P. Lorente, "Mejora en los procesos de control de aplicaciones de agroquímicos a campo," Técnica Agropecuaria, Cámara Uruguaya de Servicios Agropecuarios Congreso 100 años AIA, pp. 14-15, 2012. 